\title{
ISOLAMENTO E TRATAMENTO ACÚSTICOS EM AMBIENTE HOSPITALAR: UM ESTUDO DE CASO
}

\author{
CLÁUDIO EMANUEL PIETROBON ${ }^{1}$ \\ DORALICE APARECIDA FAVARO SOARES ${ }^{2}$ \\ PAULO FERNANDO SOARES ${ }^{2}$
}

PIETROBON, C.E.; SOARES, D.A.F.; SOARES, P.F. Isolamento e tratamento acústicos em ambiente hospitalar: um estudo de caso. Semina: Ci. Exatas/Tecnológicas, v. 16, n. 4, p. 529-535, dez. 1995.

RESUMO: Tendo em vista as opções pela qualidade total e qualidade ambiental no ambiente construído, o presente trabalho propõe uma solução para o controle de ruídos oriundos de um conjunto gerador DS1192 movido a diesel, com velocidade de $1800 \mathrm{rpm}$ e potência de $380 \mathrm{KVA}$ para instalação em ambiente hospitalar. Propõe-se o detalhamento do tratamento e do isolamento acústicos de uma casa de máquinas para abrigar o referido equipamento, limitando-se o nível de pressão sonora, externamente à edificação em $50 \mathrm{~dB}(A)$.

PALAVRAS-CHAVE: Isolamento e tratamento acústicos; Poluição sonora; Qualidade total; Qualidade ambiental.

\section{INTRODUÇÃO}

As opções pela qualidade total e ambiental, pressupõem ações integradas e interdisciplinares, nas atividades a que se destinam. O ambiente construído em quaisquer atividades humanas representa um meio de trabalho indispensável para o exercício de tais atividades. Neste contexto, ressaltam-se as influências que as variáveis energético-ambientais (SOM, LUZ, CALOR) exercem; tanto nos edifícios, incrementando o consumo energético; quanto nos usuários, causando o desconforto e a insalubridade, nos ambientes de uso e trabalho. Tendo em vista o escopo deste trabalho, expõemse alguns pontos de interesse em acústica arquitetônica, e detalham-se aspectos legais e normativos aplicados a um exame de caso.

\subsection{Condições Preliminares}

O ouvido humano é um sofisticado sensor acústico, e devido à deterioração do sistema auditivo pela prolongada exposição ao ruído, é necessário conhecer e evitar esses danos, além dos oriundos das vibrações. Som e ruído não são sinônimos. Um ruído é apenas um tipo de som, mas a reciproca não é verdadeira. O conceito de ruído é associado a um som desagradável e indesejável. O som é definido como a variação da pressão atmosférica dentro dos limites de amplitude e banda de freqüências aos quais o ouvido humano responde. Alguns limites normativos e legais, considerando que a função mais importante do sistema auditivo hu- mano é a comunicação (ouvir e entender a voz humana), afirmam que:

i) A dificuldade significativa na recepção de sons ocorre para perdas de audição maiores que $25 \mathrm{~dB}$ (valor médio nas freqüências de $500 \mathrm{~Hz}, 1.000 \mathrm{~Hz}$ e 2.000 $\mathrm{Hz}$ );

ii) A exposiçao no nível de pressão sonora abaixo de $80 \mathrm{~dB}(\mathrm{~A})$ para $90 \%$ da população, não causa dificuldades na sensação e interpretação do som; e a perda de audição por exposição a níveis acima de $80 \mathrm{~dB}(\mathrm{~A})$ depende da distribuição dos níveis com tempo de exposição e da susceptibilidade do indivíduo. Estão normalizadas e estabelecidas em forma de lei para as relações trabalhistas, visando a salubridade;

iii) As condições não trabalhistas estão normalizadas na forma de procedimentos pela Associação Brasileira de Normas Técnicas - ABNT, visando o conforto acústico

\section{METODOLOGIA}

\section{2,1. Estratégias Metodológicas}

$\mathrm{Na}$ fase de levantamento da situação de referência:

i) Vistoria ao local de implantação;

ii) Vistoria a equipamento similar em uso;

iii) Obtenção de dados acústicos de referência do equipamento em questão;

iv) Obtenção de elementos de projeto: normativos, legais e de fornecedores de materiais e componentes

1 - Universidade Estadual de Maringá - DEC -Av. Colombo, 5790 - CEP 87020-900 - Maringá - PR - Tel. (044) 226-2727, Fax (044) 222-9955. E. maii: pfsoares@brfuem.

2 - Universidade Estadual de Londrina - DCCi - Campus Universitário, CEP 86051-990 - Londrina-PR - Tel. (043) 371-4455, Fax (043) 327-6932. E. mail: doralrce@brfuet.

Semina CL Exatas/Tecn., v. 16, n. 4, p. 529-535 
de tratamento e isolação acústicos;

v) Definição da precisão e erro admissíveis.

$\mathrm{Na}$ fase de implementação:

i) Dimensionamento, e detalhamento do tratamento e isolamento acústicos para casa de máquinas do conjunto gerador DS1192 - SCANIA - 1800 rpm - 380 KVA.

\subsection{Restrições Legais e Normativas}

As restrições legais, considerando que os ruídos constituem uma das formas de poluição ambiental, pela constituição da República Federativa do Brasil, promulgada em 5/10/1988, determinam que:

i) Art. 28, VI: "É competência comum da União, dos Estados, do Distrito Federal e dos Municípios proteger o meio ambiente e combater a poluição em qualquer das suas formas."

ii) Art. 24, VI: "Compete à União, aos Estados e ao Distrito Federal legislar concorrentemente sobre florestas, caça, pesca, fauna, conservação da natureza, defesa do solo e dos recursos naturais, proteção do meio ambiente e controle da poluição."

O Código Civil brasileiro estabelece que:

i) Art. 544: "O proprietário ou inquilino de um prédio tem o direito de impedir que o mau uso da propriedade vizinha possa prejudicar a segurança, o sossego e a saúde dos que o habitam."

A CLT, nas relações do trabalho, através da Portaria $n^{\circ}$. 3.214 de 08/06/1978 do MTb. (D.O.U. de 06/07/ 1978) e a Norma Regulamentadora (N.R. 15- Atividades e operações insalubres - Cap. V - Titulo III), determinam concorrentemente limites da exposição aos ruídos, visando a salubridade e evitar danos à saúde:

QUADRO 1. Limites legais extremos à exposição de ruídos em ambientes de trabalho

\begin{tabular}{cc} 
NPS (dB(A)) Máxima exposiçāo diária permissivei \\
$\leq 85$ & 8 horas \\
115 & 7 mirutos \\
\hline
\end{tabular}

As restrições normativas nas relações não trabaIhistas, visam, além dos aspectos de salubridade, as condições de conforto acústico dentre as variáveis energético-ambientais.

Acerca do ruído urbano, segundo a NBR 10.152 (ABNT, 1987) deve-se garantir em zona hospitalar os NPS correspondentes a: $45 \mathrm{~dB}(\mathrm{a})$ durante o dia e 40 $\mathrm{dB}(\mathrm{A})$ durante a noite.

Acerca do ruído urbano, segundo a NBR 10.151 (ABNT, 1987) determina os seguintes níveis de pressão sonora:

i) Em apartamento, enfermarias, berçários e centros cir'rugicos: $35 \mathrm{~dB}(\mathrm{~A}) \leq \mathrm{NPS} \leq \mathbf{4 5} \mathrm{dB}(\mathrm{A})$;

ii) Em laboratórios e áreas para uso público: 40 $\mathrm{dB}(\mathrm{A}) \leq \mathrm{NPS} \leq \mathbf{5 5} \mathrm{dB}(\mathrm{A})$; dB(A).

iii) $\mathrm{Em}$ áreas de serviço: $45 \mathrm{~dB}(\mathrm{~A}) \leq \mathrm{NPS} \leq 55$

Acima destes níveis implica desconforto para o usuário, sem necessariamente causar dano à saúde, mas convém observar os dados referentes ã poluição sonora (OMS, 1993):

i) A exposição prolongada a ruídos com níveis acima de $85 \mathrm{~dB}$ é danosa à saúde;

ii) O limite máximo de risco é $115 \mathrm{~dB}$. Sons acima deste nível podem causar danos irreversíveis à audição em curtos períodos de exposição;

iii) A prolongada exposição a ruídos de alta intensidade - dentre os quais os agudos e os de impacto (alarmes, sirenes, britadeiras) - provoca distúrbios psicológicos e fisiológicos, tais como: enfraquecimento irreversível da audição, hipertensão arterial, aumentado batimento cardíaco, gastrites, irritabilidade, comportamento anti-social, insônia e diminuição da capacidade de raciocínio. Para dímensionamento, segue-se a NB 101 (ABNT, 1953). Em termos de normalização hospitalar, segundo a norma CIPLAN n .3 .180 publicada como Normas e Padrões de Construção e Instalação de Serviços de Saúde (Centro de Documentação do Ministério de Saúde, 1987), tem-se:

i) "Nos locais onde houver aglomeração de público, fontes de ruído ou vibração, deverão ser previstos revestimentos de acordo com a norma NB 101 da ABNT que impeçam a propagação e reverberação." [p. 33];

ii) "Quando áreas de serviço como cozinha, copa de lavagem, lavanderia, garagem, oficina ou casa de máquinas, bem como outras fontes comprovadas de ruído estiverem localizadas no mesmo bloco que as unidades de internação e tratamento dos pacientes, deverá ser previsto tratamento acústico nas paredes adjacentes por meio de material isolante de acordo com a norma NB 101 da ABNT." [0. 33];

iii) "Em hospitais com mais de 100 leitos, a fonte de emergência deverá, obrigatoriamente, ser um grupo gerador." [p. 39].

\subsection{Levantamento da Situação de Referência}

Foi efetuada vistoria e medição ao local de implantação, na data de 07/09/1994, no período vespertino, durante um feriado nacional. Procurou-se efetuar um levantamento da situação de referência para avaliar-se o mínimo ruído de fundo em condições de ocupação interna e externa ao edifício, cujos resultados encontram-se nos quadros abaixo: 
QUADRO 2. Levantamento do ruído de fundo na situação de referência

\begin{tabular}{llll}
\hline Situação & Localização & \multicolumn{1}{c}{ Condiçăo } & NPS dB(A) \\
\hline Externa & Próx. à lavanderia & Secadora em uso & 53 \\
Externa & Próx. à lavanderia & Rente à parede & 50 \\
Externa & Próx. à cabine de força & A 1,00 m de distância & 52 \\
Externa & Próx. à lavanderia & A 1,00 m de dist. janela aberta & 69 \\
Externa & Próx. à lavanderia & Idem, próx. à secadora & 69 \\
Intema & Próx. acesso c. cirürgico & Ambiente desocupado & 48 \\
Interna & Sala espera c. cirúrgico & Ambiente desocupado & 51 \\
Intema & Corredor central & Ambiente ocupado & $57-60$ \\
Intema & Berçário & Amb. ocup. cl ar condicionado & $55-60$ \\
\hline
\end{tabular}

QUADRO 3. Vistoria e medição de equipamento similar em operação

\begin{tabular}{lllll}
\hline Situação & Localizaçāo & Condiçāo & $\begin{array}{l}\text { NPS mad } \\
\text { dB(A) }\end{array}$ & $\begin{array}{l}\text { NPS } \\
\text { dB(A) }\end{array}$ \\
\hline Interna & Lateral esquerda & A 1,00 m de distäncia & 114 & $108-110$ \\
Interna & Frontal/radiador & A 1,00 m de distância & 117 & $110-112$ \\
Externa & Frontal/radiador & A 1,00 m de distância & 108 & 104 \\
Externa & Saida do escapamento & A 1,00 m de distância & 114 & $108-110$ \\
Externa & Em frente/porta aberta & A 1,00 m de distância & 105 & 104 \\
Interna & Em frente/painel de com. & A 1,00 m de distância & 114 & 110 \\
Externa & En frente/janela aberta & A 1,00 m de distância & 104 & 100 \\
\hline
\end{tabular}

Com referência aos dados do fabricante, através

1.8, tem-se:

do Manual SCANIA - Diesel Power às páginas $1.7 \mathrm{e}$

QUADRO 4. Valores de NPS do gerador DS 11 a 1800 rpm em funcionamento à potência máxima - curva 2

\begin{tabular}{|c|c|c|c|c|c|}
\hline $\begin{array}{l}\text { Freqüência } \\
(\mathbf{H z})\end{array}$ & $\begin{array}{l}\text { NPS acima } \\
\mathrm{dB}(\mathrm{A})\end{array}$ & $\begin{array}{l}\text { NPS à direita } \\
\text { dB(A) }\end{array}$ & $\begin{array}{l}\text { NPS frontal } \\
\text { dB(A) }\end{array}$ & $\begin{array}{l}\text { NPS à esq. } \\
\mathrm{dB}(\mathrm{A})\end{array}$ & $\begin{array}{l}\text { NPS médio } \\
\mathrm{dB}(\mathrm{A})\end{array}$ \\
\hline 31.5 & - & - & - & - & - \\
\hline 63 & - & 53 & $4 \mathrm{~B}$ & 52 & 50 \\
\hline 125 & 57 & 64 & 56 & 60 & 60 \\
\hline 250 & 74 & 74 & 76 & 70 & 73 \\
\hline 500 & 79 & 85 & 85 & 86 & 85 \\
\hline 1000 & 89 & 90 & 92 & 95 & 90 \\
\hline 2000 & 90 & 90 & 95 & 93 & 92 \\
\hline 4000 & 79 & 86 & 87 & 84 & 87 \\
\hline 8000 & 76 & 79 & 74 & 76 & 77 \\
\hline 16000 & 69 & 72 & 62 & 66 & 70 \\
\hline NPS médio & 97 & 100 & 101 & 101 & 100 \\
\hline
\end{tabular}


QUADRO 5. Limites de variação do ruído com a velocidade à potência total com temperatura ambiente $\leq 40^{\circ} \mathrm{C}$

Rotação (rpm)

1200

2000
Faixa NPS médio dB(A)

92-95

98-101

QUADRO 6. Análise de precisão e erro admissiveis para este estudo

\begin{tabular}{llllll}
\hline NPS & \multicolumn{2}{l}{$\begin{array}{l}\text { NPS acima NPS à direita } \\
\text { dB(A) }\end{array}$} & $\begin{array}{l}\text { NPS frontal } \\
\text { dB(A) }\end{array}$ & $\begin{array}{l}\text { NPS à esq. } \\
\text { dB(A) }\end{array}$ & $\begin{array}{l}\text { NPS médio } \\
\mathbf{d B}(\mathbf{A})\end{array}$ \\
\hline Teórico (fabricante) & 97 & 100 & 101 & 101 & 100 \\
Medido (em campo) & - & 110 & 112 & 110 & 111 \\
Erro admissivel & - & $10,0 \%$ & $10,9 \%$ & $8,9 \%$ & $11,0 \%$ \\
\hline
\end{tabular}

Face a verificação da discrepância entre os valores teóricos medidos em câmara anecóica fornecidos pelo fabricante e os obtidos por medição em condições de uso, adota-se, como precisão para este estudo, o valor de $11,0 \%$. Desta forma, os limites admissiveis de erro para o dimensionamento ficam determinados da seguinte maneira: $44 \mathrm{~dB}(\mathrm{~A}) \leq \mathrm{NPS}_{\mathrm{ext}, \mathrm{1}, 0 \mathrm{~m}} \leq 56 \mathrm{~dB}(\mathrm{~A})$, com velocidade $\leq 1800$ rpm e temperatura $\leq 40^{\circ} \mathrm{C}$.

\section{RESULTADOS, CONCLUSÃO E RECOMENDAÇÕES}

3.1. Resultados
Acerca do tratamento acústico das paredes, são propostos dois arranjos para as mesmas através da le de massa, (Beranek, 1960) com as seguintes características:

i) Configuraçâo bloco-bloco, de concreto, com $\sigma_{1}=$ $220 \mathrm{Kg} / \mathrm{m}^{2}$, espaçadas de $0,07 \mathrm{~m}$ e com recheio de placas de lä de rocha $2 ", 32 \mathrm{~kg} / \mathrm{m}^{2}$, nas dimensões de $1,20 \mathrm{~m} \times 0,60 \mathrm{~m}$;

ii) Configuração tijolo maciço-bloco com $\sigma_{1}=270$ $\mathrm{Kg} / \mathrm{m}^{2}$, para paredes externas espaçadas de $0,06 \mathrm{~m}$ e com mesmo recheio anteriormente citado.

QUADRO 7. Resultados do dimensionamento das paredes em termos de atenuação sonora, em dB

\begin{tabular}{lllllllllll}
\hline$f(\mathbf{H z})$ & $\mathbf{3 1 , 5}$ & $\mathbf{6 3}$ & $\mathbf{1 2 5}$ & $\mathbf{2 5 0}$ & $\mathbf{5 0 0}$ & $\mathbf{1 0 0 0}$ & $\mathbf{2 0 0 0}$ & $\mathbf{4 0 0 0}$ & $\mathbf{8 0 0 0}$ & $\mathbf{1 6 0 0 0}$ \\
\hline Bloco-bloco & - & 16 & 34 & 46 & 52 & 90 & 110 & $\mathbf{1 3 0}$ & $\mathbf{1 5 0}$ & 170 \\
Tijolo-bloco & 9 & 21 & 36 & 45 & 51 & 95 & 115 & $\mathbf{1 3 5}$ & $\mathbf{1 5 5}$ & 175 \\
\hline
\end{tabular}

Acerca do tratamento acústico da laje e pisco, propõem-se que a laje seja em concreto armado maciço com peso específico de $2400 \mathrm{Kg} / \mathrm{m}^{3}$ na espessura de $0,12 \mathrm{~m}$ e o piso composto de lastro de concreto simples, na espessura de $0,08 \mathrm{~m}$, revestido com borracha colada sobre a argamassa de regularização em cimento e areia no traço $1: 3$ em volume e, a base do conjunto moto-gerador, constituída de bloco de concreto armado rígido, deverá ser isolada fisicamente do piso através de junta de dilatação preenchida com material resiliente.

QUADRO 8. Resultados do dimensionamento da laje e do piso em termos de atenuação sonora, em dB

\begin{tabular}{lllllllllll}
\hline Freqüência $(\mathrm{Hz})$ & $\mathbf{3 1 , 5}$ & $\mathbf{6 3}$ & $\mathbf{1 2 5}$ & $\mathbf{2 5 0}$ & $\mathbf{5 0 0}$ & $\mathbf{1 0 0 0}$ & $\mathbf{2 0 0 0}$ & $\mathbf{4 0 0 0}$ & $\mathbf{8 0 0 0}$ & $\mathbf{1 6 0 0 0}$ \\
\hline Concr. armado & 38 & 38 & 38 & 38 & 48 & 58 & 68 & 78 & 88 & 98 \\
\hline
\end{tabular}


Com referência às portas acústicas, serão duas, dimensionadas em série, com 1,61 m x 2,20m.

QUADRO 9. Resultados do dimensionamento das portas em termos de atenuação sonora, em dB

\begin{tabular}{llllllllll}
\hline Freqüēncia (Hz) & 63 & 125 & 250 & 500 & 1000 & 2000 & 4000 & 8000 & 16000 \\
\hline Duas portas em série fechadas & 40 & 48 & 53 & 59 & 63 & 65 & 65 & 65 & 65 \\
\hline
\end{tabular}

Em relação aos atenuadores de ruído, dimensionados por absorção sonora (Gerges, 1992), a entrada de arfrio, será constituída de uma caixa de concreto armado com dimensão interna de $1,22 \mathrm{~m} \times 1,96 \mathrm{~m}$, contendo 15 linhas de placas absorventes tipo baffe de $0,80 \mathrm{~m} \times 1,20 \mathrm{~m}$, de espessura $40 \mathrm{~mm}$, fixadas em estirantes metálicos transversais na base e no topo da mesma, compondo 5 séries longitudinais com um comprimento mínimo de $4,00 \mathrm{~m}$, totalizando 140 placas. As extremidades do duto deverão ser revestidas com tela metálica para evitar o acesso de insetos e pequenos animais. Deve-se garantir uma área mínima de ventilação de $1,00 \mathrm{~m}^{2}$.

QUADRO 10. Resultados do dimensionamento da entrada de ar frio em termos de atenuação sonora, em dB

\begin{tabular}{lllllllll}
\hline Freqüência (Hz) & 63 & 125 & 250 & 500 & 1000 & 2000 & 4000 & 8000 \\
\hline Atenuador de entrada & - & 38 & 46 & 53 & 61 & 61 & 61 & - \\
\hline
\end{tabular}

Para a saída de ar quente, adotou-se a mesma geometria de seção transversal ã exceção do comprimento que será $5,10 \mathrm{~m}$, reservando-se $1,10 \mathrm{~m}$ para a câmara de fumaça no início do duto. Ressalta-se que a ligação do radiador com o duto deve ser efetuada em material resiliente e flexível, devido à necessidade de direcionar o ruído para o duto e adequar a ligação devido aos amortecedores anti-vibratóríos da base do conjunto moto-gerador. Deve-se garantir, também, uma seção mínima livre para ventilação de $1,00 \mathrm{~m}^{2}$. Os resultados da atenuação, em dB, são idênticos aos do quadro 10.

Para a saída de gases, adotaram-se tubos de descarga com diâmetros nominais crescentes de 4", 6" e 8", interligados com silenciadores. Os tubos de descarga foram revestidos com tubos bipartidos de silicato de cálcio nos diâmetros internos nominais de 4", 6" e 8" com espessura de $50 \mathrm{~mm}$, visando isolação térmica e acústica. Sobre a laje deverá ser executado um duto de concreto armado com dimensão interna de $1,96 \mathrm{~m} \mathrm{x}$ $1,22 \mathrm{~m}$, sendo as suas paredes internas revestidas com absorvedor acústico, espessura de $35 \mathrm{~mm}$, em placas de $1,0 \mathrm{~m} \times 1,0 \mathrm{~m}$.

Para o ambiente da sala de máquinas, visando a melhoria da absorção do ruído gerado internamente foram utilizados absorvedores acústicos a saber: paredes (revestimento absorvente com espessura de $35 \mathrm{~mm}$, em placas de $1,0 \mathrm{~m} \times 1,0 \mathrm{~m}$, coladas sobre a superfície); piso \{revestimento absorvente, em borracha, colado sobre a superfície); teto (forro absorvente com 1" de espessura, em placas de $1,25 \mathrm{~m} \times 0,60 \mathrm{~m}$ assentadas sobre guias de alumínio rentes à laje de cobertura).

Para o ambiente da sala de comando adotou-se a mesma especificação anterior e os resultados do dimensionamento apresentam-se a seguir:

QUADRO 11. Resultados do dimensionamento da atenuação por absorçāo sonora, em dB

\begin{tabular}{lllllllll}
\hline Freqüéncia $(\mathbf{H z})$ & 63 & 125 & 250 & 500 & 1000 & 2000 & 4000 & 8000 \\
\hline Sala de máquinas & - & 3 & 8 & 11 & 12 & 11 & 8 & - \\
Sala de comando & - & 2 & 8 & 11 & 12 & 12 & 9 & - \\
\hline
\end{tabular}


Ressalta-se que o presente tratamento visa diminuir as condições agressivas dos níveis de ruído tendo em vista o nível de exposição aos ruídos dos funcionários que operarão o grupo gerador. Entretanto, salienta-se a necessidade de utilização de EP! (Equipamento de Proteção Individual) - Protetor Auricular, sempre que se necessitar acesso a estes ambientes.

Para o tratamento anti-vibratório, adotou-se um sistema de isolação composto por um conjunto de seis amortecedores inteiramente metálicos, à base de molas helicoidais com aimofada em aço inoxidável interna, atuando a baixas freqüências $(7$ a $9 \mathrm{~Hz}$ ), distribuídos sob o conjunto moto-gerador, simetricamente em relação ao centro de gravidade.

Finalmente, para o tratamento térmico, segundo indicações do fabricante, visando ã garantia do desempenho do conjunto moto-gerador, deve-se limitar a temperatura interior da sala de máquinas entre 17 e $40^{\circ} \mathrm{C}$. Para tanto simulou-se o desempenho térmico da sala de máquinas com os seguintes parâmetros de operação: taxa de ventilação de 22,4 renovações por hora, supondo-se o caso crítico (dados médios e sazonais do clima de Maringá de uma série histórica de 10 anos, adotando-se o critério de média aritmética simples entre as temperaturas absolutas e médias) e considerando-se 6.000 watts de geração de calor devido ao funcionamento do grupo-gerador por 24 horas consecutivas. Foi realizada a simulação do desempenho térmico e ressalta-se que é necessário efetuar a pintura externa das paredes e telhas na cor branca. A temperatura mínima será de $17^{\circ} \mathrm{C}$ no caso de inverno e só ocorrerá às 10 horas da manhã, e a máxima temperatura atingirá no verão $38^{\circ} \mathrm{C}$ entre 14 horas e 16 horas.

\subsection{Conclusão}

Com o tratamento e as especificações propostas, pretendeu-se atingir os valores de nível de pressão sonora descritos no item 2.3. Para a garantia dos níveis equivalentes aos do ruído urbano previstos no item 2.2. da normalização, na vizinhança deve-se garantir o máximo de área gramada no entorno da edificação e executar um muro divisório com altura mínima de 4,0m com forração vegetal.

\subsection{Recomendações}

Para a garantia dos níveis de pressão sonora definidos anteriormente, deve-se, quanto ã execução das paredes, pisos e lajes, evitar o surgimento de quaisquer tipos de fissuras que venham a comprometer o desempenho acústico previsto para o componente; quanto às ligações externas com o pavimento, devem ser executadas através de juntas fiexíveis de baixa rigidez, com o objetivo de não transmitir vibrações ás tubulações ou à estrutura da edificação. A base do conjunto moto-gerador deverá ter rigidez suficiente e isolamento físico do restante da estrutura da edificação.

Quanto às juntas previstas na estrutura, a parede dupla deve ter isolamento físico entre as paredes internas (blocos) e as paredes externas (tijolos); as juntas das placas de concreto devem ser executadas evitando a saída de ruidos e penetração de umidade através de bolsões de ar e fissuras oriundas da má execução. A vedação e instalação das portas acústicas, bem como dos amortecedores anti-vibratórios do conjunto motogerador devem seguir rigorosamente as instruções dos fabricantes.

Para assegurar o desempenho térmico, deve-se: garantir a área útil de ventilação nos dutos em $1 \mathrm{~m}^{2}$; executar e manter as pinturas na cor branca nas paredes externas e telhamento. 


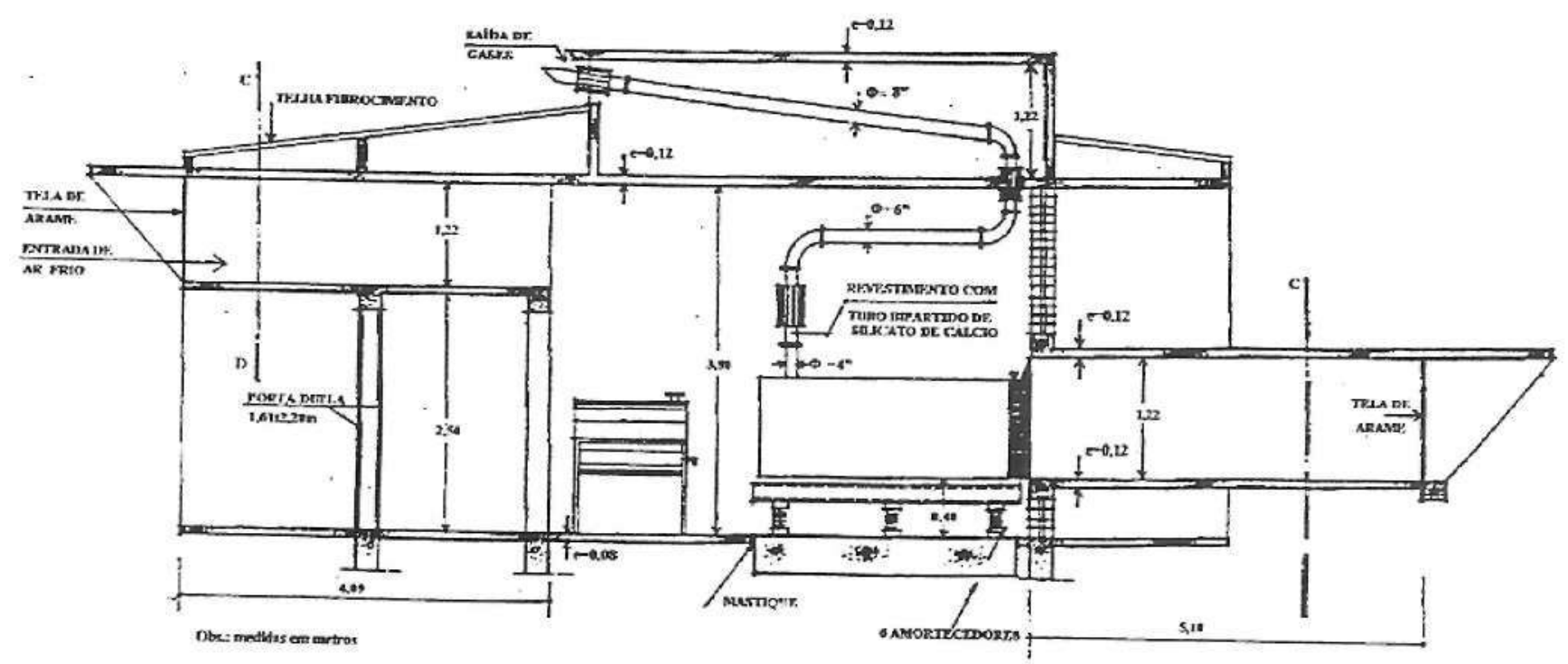

FIGURA 1. Corte esquemático da solução proposta

PIETROBON, CE.; SOARES, D.A.F.; SOARES, P.F. Isolation and acoustic treatment In a hospital environment: a case study. Semina: Ci. Exatas/Tecnológicas, v. 16, n. 4, p. 529-535, Dec. 1995.

ABSTRACT: Having in mind the total and environmental quality into the building environment, this present work proposes a solution to control de noise derived from a diesel machine-model DS1192, with approximately $1800 \mathrm{rpm}$ speed and approximately $380 \mathrm{KVA}$ power, placed in a hospital building. It also proposes details for the house machine building acoustic treatment and isolation, limiting the external sound pressure level, to $50 \mathrm{~dB}(A)$.

KEY-WORDS: Isolation and acoustic treatment; sound pollution; total quality; environmentaí quality.

\section{REFERÊNCIAS BIBLIOGRÁFICAS}

ACÚSTICA \& VIBRAÇŌES. Absorção Acústica de Materiais de Acabamento de Construçāo. Săo Paulo: SOBRAC, n. 12, jun. 1993.

ASSOCIAÇĀO BRASILEIRA DE NORMAS TÉCNICAS. Rio de Janeiro. Avaliaçäo do ruido em áreas habitadas visando o conforto da comuridade, NBR 10.151. Rio de Janeiro, 1987.

Rỉo de Janeiro. Niveis de nuido para conforto acústico, NBR 10.152. Rio de Janeiro, 1987.

Rio de Janeiro. Norma para tratamento acústico em recintos fechados, NB 101. Rio de Janeiro, 1971.

BERANEK, Leo L. Acoustics. New York: McGraw-Hitl, 1954.

1960.

Noise Reduction. New York: McGraw-Hill,

BRASIL. Portaria $n^{\circ} .3214$, de 08 de junho de 1978. Aprova as Normas Regulamentadoras - NR - do Capitulo V do Título II da Consolidaçāo das Leis do Trabalho, relativo à Segurança e Medicina do Trabalho. Diário Oficial da Uniăo, Brasilia, 06 jul. 1978 (suplememo).

Resolyçấo n. 1, de 11 de fevereiro de 1993.
Estabelece, para os devidos automotores nacionais e importados, exceto motocicletas, motonetas, ciclomotores, bicicleta com motor auxiliar e veiculos assemelhados, limites máximos de ruido com veículos em aceleração e na condiçâo parado. Diário Oficial da Uniäo, Brasília, 15 fev. 1993.

Resoluçäo $n^{\circ}$. 2, de 11 de fevereiro de 1993. Estabelece, para motocicletas, motonetas, triciclos, ciclomotores, bicicleta com motor auxiliar e veiculos assemelhados, nacionais e importados, limites máximos de ruido com veículos em aceleração e na condição parado. Diărio Oficial da Uniāo, Brasília, Brasifia, 23 dez. 1977.

Lei $n^{\circ}$. 6.514, de 22 de dezembro de 1977. Altera o Capitulo V do Título II da Consolidaçäo das Leis do Trabalho, relativo à Segurança e Medicina do Trabalho. Diário Oficial da Uniâo, Brasillia, 23 dez. 1977.

EGAN, David M. Concepts in Architectural Acoustics. New York: McGraw-Hill, 1972.

GERGES, Samir N.Y. Ruido: fundamentos e controle. Florianópolis: Centro Brasileiro de Segurança e Saúde Industrial, 1992.

MiŇANA, José Pérez. Compendio Práctico de Acústica. Barcelona: Editorial Labor, 1969. 